\title{
VARIAÇÕES DA FISIONOMIA, DIVERSIDADE E COMPOSIÇÃO DE GUILDAS DA COMUNIDADE ARBÓREA EM UM FRAGMENTO DE FLORESTA SEMIDECIDUAL EM LAVRAS, MG ${ }^{1}$
}

\author{
Yule Roberta Ferreira Nunes ${ }^{2}$ \\ Andrea Vita Reis Mendonça ${ }^{2}$ \\ Luciana Botezelli ${ }^{2}$ \\ Evandro Luiz Mendonça Machado ${ }^{2}$ \\ Ary Teixeira de Oliveira-Filho ${ }^{2}$
}

Recebido em 12/07/2001. Aceito em 11/07/2002

\begin{abstract}
RESUMO - (Variações da fisionomia, diversidade e composição de guildas da comunidade arbórea em um fragmento de floresta semidecidual em Lavras, MG). Este estudo teve como objetivo analisar diferenças na estrutura fisionômica, na diversidade de espécies e na composição de guildas da comunidade arbórea de setores de um fragmento de floresta semidecidual que se encontram em diferentes fases de regeneração e graus de exposição à borda. O fragmento, que possui 5,8ha de área e está situado no município de Lavras, MG (2113'40"S, $44^{\circ} 57^{\prime} 50^{\prime \prime} \mathrm{W}$ ), foi dividido em quatro setores e submetido a um censo das árvores com DAP $\geq 5 \mathrm{~cm}$. Os setores foram comparados quanto à densidade, área basal, distribuição de alturas e de diâmetros, diversidade de espécies e freqüência de árvores por guildas de regeneração, estratificação e dispersão. Os setores menos perturbados no passado, Interior Alto e Borda Alta, apresentaram maiores áreas basais e menores densidades de árvores (sobretudo das de menor tamanho), maiores proporções de árvores de espécies clímax tolerantes à sombra e de espécies de grande porte que os mais perturbados, Interior Baixo e Borda Baixa. Estas diferenças realçam que os setores Altos estão em uma fase mais avançada do processo de regeneração. Os setores de Borda tiveram maior frequiência de indivíduos de espécies com dispersão anemocórica que os de Interior. Cada setor de Borda teve maior diversidade de espécies que seu parceiro de Interior. Concluiu-se que os parâmetros analisados podem, em conjunto, servir como bons avaliadores da severidade da perturbação sofrida no passado, da fase regenerativa atual e do efeito borda.
\end{abstract}

Palavras-chave - guildas de dispersão, guildas de estratificação, guildas de regeneração, distúrbios, fragmentação

1 Este trabalho integra o Subprojeto Estratégias para conservação e manejo da biodiversidade em fragmentos de florestas semidecíduas executado pela EMBRAPA Recursos Genéticos, a Universidade Federal de Lavras (UFLA) e a Universidade de Brasília (UnB) e o Projeto de Conservação e Utilização Sustentável da Diversidade Biológica Brasileira (PROBIO) supervisionado pelo Ministério do Meio Ambiente (MMA), gerido pelo Conselho Nacional de Desenvolvimento Científico e Tecnológico (CNPq) e apresentado pelo governo brasileiro ao GEF/BIRD

2 Departamento de Ciências Florestais, Universidade Federal de Lavras, CEP 37200-000, Lavras, MG, Brasil 


\begin{abstract}
Variations in tree community physiognomy, diversity, and species guild composition of a fragment of tropical semideciduous forest in Lavras, south-eastern Brazil). The purpose of the present study was to analyze differences in physiognomic structure, species diversity, and species guild composition of the tree community of different sectors of a tropical semideciduous forest fragment. Each sector varies in terms of regeneration phase and exposure to the forest edges. The forest fragment, which has an area of 5.8ha and is situated in Lavras municipality, Minas Gerais State, Brazil (21 $13^{\prime} 40^{\prime \prime}$ ' $\left.; 44^{\circ} 57^{\prime} 50^{\prime \prime} \mathrm{W}\right)$, was classified into four sectors and underwent a census of all trees with $\mathrm{dbh} \geq 5.0 \mathrm{~cm}$. The sectors were compared for tree density and basal area, distribution of heights and diameters, species diversity, and frequency of trees per regeneration, dispersal, and stratification guilds. The sectors that were more lightly disturbed in the past, Tall Interior and Tall Edge, showed higher tree basal areas, lower tree densities (particularly for smaller size classes), and higher proportions of individuals of shade-tolerant tree species and tall tree species, than the more heavily disturbed sectors, Low Interior and Low Edge. These differences indicate that the Tall sectors are at a more advanced regeneration phase. The Edge sectors had higher frequency of trees of anemochorous species than the Interior sectors. Each Edge sector had higher species diversity than its Interior partner. It was concluded that the analyzed parameters may serve as efficient means of assessment of past disturbance severity as well as of present regeneration phase and edge effects.
\end{abstract}

Key words - Dispersal guild, disturbance, fragmentation, regeneration guild, stratification guild

\section{Introdução}

Nas florestas tropicais é possível identificar áreas em diferentes fases de regeneração formando mosaicos florestais. Whitmore (1990) reconheceu três fases na regeneração de uma floresta tropical: a fase de clareira, a fase de regeneração e a fase madura. Assim, os setores que compõem estes ambientes se diferenciam em relação à composição de espécies e, conseqüentemente, apresentam variações nos padrões de regeneração, estruturação e dispersão. Segundo Pires-O'Brien \& O'Brien (1995) o ambiente da floresta tropical é heterogêneo no espaço e no tempo e a formação de clareiras é fator importante na manutenção desta heterogeneidade. Além disto, a paisagem de uma área é composta por um complexo de unidades interativas (Metzger 1999), onde a intensidade dos distúrbios causados determina a velocidade de regeneração deste ambiente (Whitmore 1990).

Os fragmentos florestais merecem atenção especial, pois além de estarem sujeitos aos eventos naturais que promovem a formação de ambientes em diferentes fases de regeneração, também estão mais susceptíveis à ação antrópica e ao efeito de borda. De acordo com Kapos (1989) e Laurance \& Yensen (1991), os estudos sobre as consequiências da fragmentação para as florestas tropicais, particularmente os realizados na Amazônia Central (Lovejoy et al. 1983; 1984; 1986; Lovejoy \& Bierregaard 1990) e no Panamá (Williams-Linera 1990a; 1990b), indicam a ocorrência de alterações na estrutura e dinâmica da comunidade arbórea, principalmente próximo às bordas recentemente criadas, onde mudanças no microclima, principalmente no regime de luz e balanço de água no solo, desencadeiam mudanças biológicas drásticas.

A quase totalidade da cobertura florestal primitiva da Região do Alto Rio Grande, no centro-sul do estado de Minas Gerais, foi reduzida a remanescentes esparsos, a grande maioria com menos de 10ha (Oliveira-Filho et al. 1994). Fragmentos como estes sofrem, freqüentemente, interferências inerentes ao processo de fragmentação, como o efeito de borda e o isolamento da área (Hunter 1996), e também perturbações antrópicas, como a retirada seletiva de madeira e o fogo. Desta forma, torna-se urgente a necessidade de se avaliar a diversidade biológica neles contida, bem como compreender a organização espacial da comunidade de plantas no seu interior e a direção das mudanças nos processos ecológicos, o que permitirá traçar práticas de manejo com vistas à conservação de sua diversidade 
biológica a longo prazo.

O presente trabalho teve como objetivo fundamental reconhecer padrões ecológicos gerais da comunidade arbórea de setores de um fragmento de floresta semidecídua que se encontram em diferentes fases de regeneração pós-distúrbio e graus de exposição à borda $\mathrm{e}$ analisar a possibilidade de se utilizarem estes padrões para se avaliar o estádio regenerativo e o efeito borda. Desta forma, os setores foram comparados nos aspectos estrutura fisionômica, diversidade de espécies e composição de guildas de regeneração, estratificação e dispersão.

\section{Material e métodos}

Área de estudo - Este estudo foi realizado na Reserva Florestal da Universidade Federal de Lavras (UFLA), a qual está situada no município de Lavras (MG), nas coordenadas $21^{\circ} 13^{\prime} 40^{\prime \prime}$ S e $44^{\circ} 57^{\prime} 50^{\prime \prime}$ W e a uma altitude de $925 \mathrm{~m}$, e é constituída de um fragmento de floresta estacional semidecidual montana, com área de 5,8ha (Oliveira-Filho et al. 1994). O clima da região é do tipo Cwb (Köppen), com verões brandos e invernos secos. As médias anuais de precipitação e temperatura são de $1.529,5 \mathrm{~mm}$ e $19,4^{\circ} \mathrm{C}$, respectivamente, sendo o período de maior concentração de chuvas de novembro a março (DNMet 1992). De acordo com o sistema brasileiro de classificação (EMBRAPA 1999), os solos são Latossolos Vermelhos Distroférricos típicos, bem drenados e com textura muito argilosa a argilosa. Desde a década de 1920 este fragmento não sofreu corte raso e manteve-se aproximadamente com os mesmos limites, exceto na borda leste, onde houve corte nos anos 70. Entretanto, até 1986, quando a mata foi declarada de preservação permanente, ocorreu retirada seletiva de madeira e aberturas de trincheiras para estudos de perfil de solo (Oliveira-Filho et al. 1997).

Fonte de dados - Em 1986, a área da Reserva Florestal da UFLA foi dividida em 126 parcelas de $400 \mathrm{~m}^{2}(20 \times 20 \mathrm{~m})$, cobrindo $86,5 \%$ da área e totalizando 5,04ha de área (Fig. 1). Em 1987, foi realizado o primeiro inventário censitário de todos os indivíduos arbóreos com DAP (diâmetro a altura do peito $=1,30 \mathrm{~m}$ do solo) $\times 5 \mathrm{~cm}$, os quais foram marcados com plaquetas de alumínio. Para cada árvore, foram registrados o nome da espécie, o PAP (perímetro a altura do peito) e a altura total. Os resultados do primeiro inventário encontram-se em Oliveira-Filho et al. (1994). Seguiram-se os inventários de 1992 (Oliveira-Filho et al. 1997), 1996 (Pulz 1998; Chagas et al. 2001) e 2001, registrando-se a ocorrência de mortes, incorporando-se os recrutas e registrando-se as novas medidas das árvores sobreviventes. $\mathrm{O}$ presente trabalho utiliza os dados do inventário de 2001.

No estudo da dinâmica da comunidade arbórea da Reserva Florestal da UFLA no período compreendido entre 1987 e 1992, Oliveira Filho et al. (1997) dividiram o fragmento em quatro setores fisionômicos Borda Baixa, Borda Alta, Interior Baixo e Interior Alto - baseados nas diferenças de altura do dossel e da densidade do sub-bosque (Fig. 1). De acordo com os autores acima, parte do Interior Alto está localizado em condições de borda provavelmente devido à remoção local de parte floresta nos anos 70. Análises multivariadas dos parâmetros de dinâmica da comunidade e composição de espécies confirmaram a consistência da classificação (Oliveira Filho et al. 1997). As diferenças foram atribuídas ao regime de distúrbios no passado, tendo os setores Baixos sofrido distúrbios mais severos que os Altos, e ao efeito borda, sendo que os setores de Borda apresentavam subbosque e massa de lianas mais densos que os de Interior. Estes mesmos quatro setores foram utilizados em análises comparativas no presente estudo.

Classificação ecológica das espécies - Para comparar os diferentes setores da floresta quanto ao perfil de estratégias ecológicas de suas 


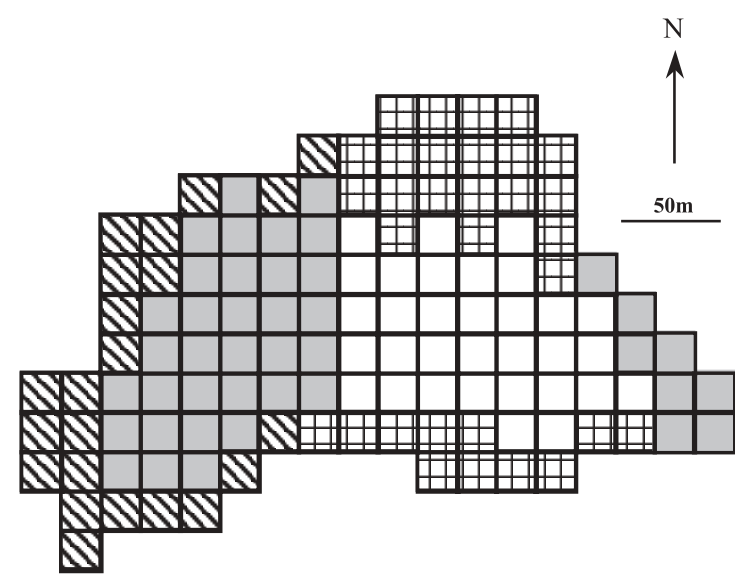

Figura 1. Distribuição das 126 parcelas $(20 \times 20 \mathrm{~m})$ utilizadas no inventário censitário da comunidade arbórea da Reserva Florestal da UFLA, Lavras, MG, mostrando a classificação das parcelas em quatro setores feita com base nas características fisionômicas da vegetação. Setores do fragmento: $\boxplus$ Borda Baixa; $\mathbb{Z}$ Borda Alta; $\square$ Interior baixo; $\square$ Interior Alto.

espécies arbóreas, estas foram classificadas em três sistemas de guildas de acordo com as estratégias de regeneração, estratificação e dispersão (veja Apêndice).

As espécies foram classificadas nas guildas de regeneração definidas por Swaine \& Whitmore (1988), sendo: (1) pioneiras, aquelas que necessitam de luz direta para germinar e se estabelecer; (2) clímax exigentes de luz, aquelas cujas sementes conseguem germinar nas condições de sombra do sub-bosques, embora os imaturos necessitem de luz abundante para crescer e atingir o dossel; e (3) clímax tolerantes à sombra, aquelas que germinam e conseguem crescer nas condições de sombra do sub-bosque, atingindo a maturidade sob o dossel ou no dossel da floresta, conforme a espécie.

A classificação das espécies em guildas de estratificação foi baseada na altura normalmente alcançada pelas árvores na maturidade e utilizando as mesmas categorias e intervalos definidos por Oliveira-Filho et al. (1997): (1) pequenas, espécies com altura entre 2,0 a 7,9m; (2) médias, entre 8,0 a 17,4m alt.; e (3) grandes, aquelas que ultrapassaram $17,5 \mathrm{~m}$ alt.
Quanto às guildas de dispersão, as espécies foram classificadas, de acordo com Van der Pijl (1982), nas categorias: (1) anemocóricas, aquelas cujas sementes são disseminadas pelo vento; (2) zoocóricas, aquelas que apresentam características que indicam que a dispersão de sementes é feita por animais; e (3) autocóricas, aquelas que dispersam suas sementes pela gravidade ou por deiscência explosiva.

Para a classificação das espécies nos três sistemas de guildas, foram utilizadas informações sobre a biologia das espécies encontradas na literatura (Morellato \& LeitãoFilho 1992; Lorenzi 1992, 1998; Oliveira-Filho et al. 1995; 1997; Barroso et al. 1999) e acrescidas do conhecimento dos próprios autores.

Análise dos dados - Os quatro setores do fragmento foram comparados para as seguintes características de estrutura fisionômica: densidade e área basal das árvores e distribuição de tamanhos (diâmetro e altura). As diferenças de densidade e área basal entre os setores foram avaliadas por Análises de Variância e testes de Tukey-Kramer, aplicados após o teste de Bartlett para normalidade (Zar 1996). Para isto, considerou-se um delineamento em blocos com número de parcelas diferentes (Borda Baixa, 31; Borda Alta, 22; Interior Baixo, 32; e Interior Alto, 41 parcelas) e os quatro setores como tratamentos. As análises foram realizadas no programa SYSTAT/SYSGRAF, versão 5.0, e feitas com propósito basicamente instrumental, uma vez que o universo comparado não atende aos requisitos de amostragem (os setores são divisões de um censo) e de independência e aleatoriedade das repetições (as parcelas são adjacentes).

Foram preparadas distribuições de densidade de árvores por classes de diâmetro e altura para os quatro setores do fragmento, empregando intervalos de classe com amplitudes crescentes para compensar o forte decréscimo da densidade nas classes de tamanho maiores (Botrel et al. 2002). Foi adotada a 
densidade de árvores por hectare ao invés da frequiência por classes porque os setores tinham áreas desiguais.

Foram calculados, para cada setor, o índice de diversidade de espécies de Shannon, $H^{\prime}$, e a equabilidade de Pielou, $J$ ' (Krebs 1989). Os índices de diversidade de Shannon dos setores foram comparados pelo teste de $t$ de Hutcheson (Zar 1996). As comparações foram feitas aos pares porque o teste de $t$ de Hutcheson é o único disponível para comparações estatísticas entre valores de $H^{\prime}$.

Para verificar se a distribuição das frequiências de indivíduos arbóreos nas guildas de regeneração, estratificação e dispersão era independente do setor do fragmento foi aplicado o teste de qui-quadrado para tabelas de contingência (Zar 1996). O mesmo foi feito em tabelas de contingência cruzando a freqüência de espécies (e não indivíduos) nas guildas de regeneração $\times$ dispersão, regeneração $\times$ estratificação e estratificação $\times$ dispersão. Devido ao pequeno número de espécies, as guildas 'pequeno porte' e 'autocórica' foram fundidas às guildas 'médio porte' e 'anemocórica', resultando, respectivamente, nas guildas 'dispersão primária abiótica' e 'sub-bosque'.

\section{Resultados e discussão}

Estrutura fisionômica da comunidade arbórea - A densidade média e a área basal média diferiram significativamente entre os quatro setores da floresta (Tab. 1). Não houve diferença significativa entre os dois setores Altos e os dois setores Baixos da floresta, tanto para densidade média como para área basal média. Nos setores Baixos, as densidades médias são maiores e as áreas basais médias menores que nos setores Altos, embora a densidade do Interior Baixo não tenha diferido significativamente da densidade dos demais setores. Estas diferenças sugerem uma comunidade mais jovem nos setores Baixos, com maior densidade de árvores mais finas, ao passo que, nos setores Altos, a maior densidade de árvores mais grossas sugere estádio de regeneração mais avançado.

É bem conhecido que a densidade e a área basal médias das florestas tropicais variam muito com as condições de solos, água e luz bem como entre estádios de regeneração. Geralmente, florestas maduras apresentam maior número de árvores com áreas basais grandes, enquanto aquelas em estádios mais iniciais de regeneração, formam grandes adensamentos de árvores finas (Uhl \& Murphy 1981; Parthasarathy 1999). Como, no presente caso, os solos e a topografia são muito homogêneos, as diferenças entre os setores Baixos e Altos provavelmente devem-se às perturbações mais severas sofridas pelos primeiros no passado, resultando em um estádio regenerativo mais jovem. Outra fonte de variação potencial de habitats, o efeito borda, pode incrementar localmente área basal e densidade devido à maior abundância de luz (Murcia 1995). Contudo, não houve diferenças claras entre setores de Interior e de Borda, embora a maior densidade de árvores finas na Borda Baixa provavelmente tenha contribuído para que este se diferenciasse significativamente dos setores Altos, ao contrário do Interior Baixo.

A estrutura de tamanhos de uma comunidade de plantas é o resultado da ação de fatores bióticos e abióticos que atuam sobre as populações, sendo também um dos indicativos do status de regeneração da vegetação (Clark 1994; Hutchings 1997; Condit et al. 1998). A Figura 2 mostra as distribuições de tamanhos das árvores nos quatro setores do fragmento florestal expressas como médias, mais desvios padrão da densidade de árvores nas classes de altura e diâmetro. A distribuição da densidade das árvores com DAP $\geq 5 \mathrm{~cm}$ por classe de altura (Fig. 2A) mostra diferenças marcantes apenas na classe de $>5$ a $10 \mathrm{~m}$ alt., que apresentou maior densidade de árvores nos dois setores Baixos, indicando maior concentração de árvores mais baixas que nos setores Altos. Além disso, nota- 
Tabela 1. Densidade, área basal, número de indivíduos, número de espécies (S), índice de diversidade de Shannon $\left(H^{\prime}\right)$ e equabilidade de Pielou $(J)$ obtidos para as árvores $\geq 5 \mathrm{~cm}$ de DAP inventariadas nos quatro setores da Reserva Florestal da UFLA, Lavras, MG. Os valores de densidade e área basal são médias \pm desvios padrão das N parcelas de cada um dos quatro setores, comparados por análises de variância. As médias seguidas de letras diferentes indicam diferenças significativas em testes de Tukey $(P<0,05)$.

\begin{tabular}{|c|c|c|c|c|c|c|c|}
\hline Setores & $\mathrm{N}$ & $\begin{array}{c}\text { Densidade } \\
\left(\text { árvores ha' }{ }^{-1}\right)\end{array}$ & $\begin{array}{c}\text { Área basal } \\
\left(\mathrm{m}^{2} \mathrm{ha}^{-1}\right)\end{array}$ & $\begin{array}{l}\text { Núm. } \\
\text { indiv. }\end{array}$ & $S$ & $\begin{array}{c}H^{\prime} \\
\text { (nats/indiv.) }\end{array}$ & $J^{\prime}$ \\
\hline Total & 126 & $1291,1 \pm 271,4$ & $22,9 \pm 6,0$ & 6507 & 153 & 3,67 & 0,73 \\
\hline Interior Alto & 41 & $1208,5 \pm 268,4 b$ & $24,6 \pm 5,2 a$ & 1982 & 109 & 3,49 & 0,74 \\
\hline Borda Alta & 22 & $1177,3 \pm 267,5 b$ & $27,7 \pm 4,8 a$ & 1036 & 109 & 3,75 & 0,80 \\
\hline Interior Baixo & 32 & $1307,0 \pm 201,9 a b$ & $19,0 \pm 4,9 b$ & 1673 & 65 & 3,26 & 0,78 \\
\hline $\begin{array}{l}\text { Borda Baixa } \\
\text { ANOVA }\end{array}$ & 31 & $\begin{array}{c}1464,6 \pm 261,9 a \\
F=7,94 \\
P<0,0001\end{array}$ & $\begin{array}{c}21,4 \pm 5,6 \quad b \\
F=14,38 \\
P<0,0001\end{array}$ & 1816 & 100 & 3,43 & 0,74 \\
\hline
\end{tabular}

se também maior densidade de árvores da classes de altura subsequientes (>10 a $15 \mathrm{~m}$ e $>15$ a 20m) nos setores Altos, embora a diferença seja menos pronunciada. A distribuição de árvores em classes de diâmetro oferece vantagens na avaliação da estrutura de tamanhos porque o estiolamento de árvores mais baixas não interfere tanto nas comparações. A distribuição da densidade das árvores em classes de diâmetro (Fig. 2B) mostra que os setores Baixos da floresta apresentaram maior densidade de árvores que os setores Altos na menor classe de diâmetro $(>5 \mathrm{a} 10 \mathrm{~cm})$, enquanto o contrário foi observado para os setores Altos, que tiveram maior densidade de árvores mais grossas (>20 a $40 \mathrm{~cm})$. Desta forma, a distribuição das árvores em classes de altura e diâmetro nos diferentes setores da floresta confirmam os padrões sugeridos pela densidade e área basal médias. As áreas que sofreram perturbações mais severas no passado possuem maior densidade de árvores finas e baixas, caracterizando estádio de regeneração inicial. Já os setores que sofreram distúrbios mais leves no passado apresentaram maior densidade de árvores altas e grossas, indicando estádio regenerativo mais avançado.

Diversidade - A diversidade de determinada área pode ser medida, de forma simplificada, por índices de diversidade, como o de Shannon $\left(H^{\prime}\right)$, que combinam o número de espécies que ocorrem na amostra, ou riqueza, com a distribuição de suas respectivas abundâncias, ou equabilidade (Gaston 1996). A riqueza variou pouco, de 100 a 109 espécies, entre os dois setores Altos e a Borda Baixa, mas o Interior Baixo destacou-se pela menor riqueza: apenas 65 espécies (Tab. 1). Como a equabilidade do Interior Baixo foi das mais elevadas $\left(J^{\prime}=0,78\right)$, sua baixa riqueza de espécies foi, certamente, a principal responsável pelo seu índice de Shannon ( $H^{\prime}=3,26$ nats/indivíduo) ter sido o mais baixo e também significativamente inferior aos dos demais setores (comparações pelo teste de $t$ de Hutcheson com Borda Baixa: $t=4,25, P<0,001$; Borda Alta: $t=-10,70, P<0,001$; Interior Alto: $t=-5,92 ; P<0,001)$. No outro extremo, o maior $H$ ' foi encontrado para a Borda Alta (3,75 nats/ indivíduo), o qual foi significativamente superior aos do Interior Alto $(t=5,37, P<0,001)$ e Borda Baixa $(t=-6,54, P<0,001)$. Contribuíram para isto tanto o maior valor de equabilidade $\left(J^{\prime}=\right.$ 0,78 ) como a maior riqueza de espécies, se considerarmos o menor tamanho do setor Borda Alta, tanto em área como em número de indivíduos. Apenas o Interior Alto e a Borda Baixa não diferiram estatisticamente nos seus valores de $H^{\prime}(t=-1,44, P>0,05)$. 

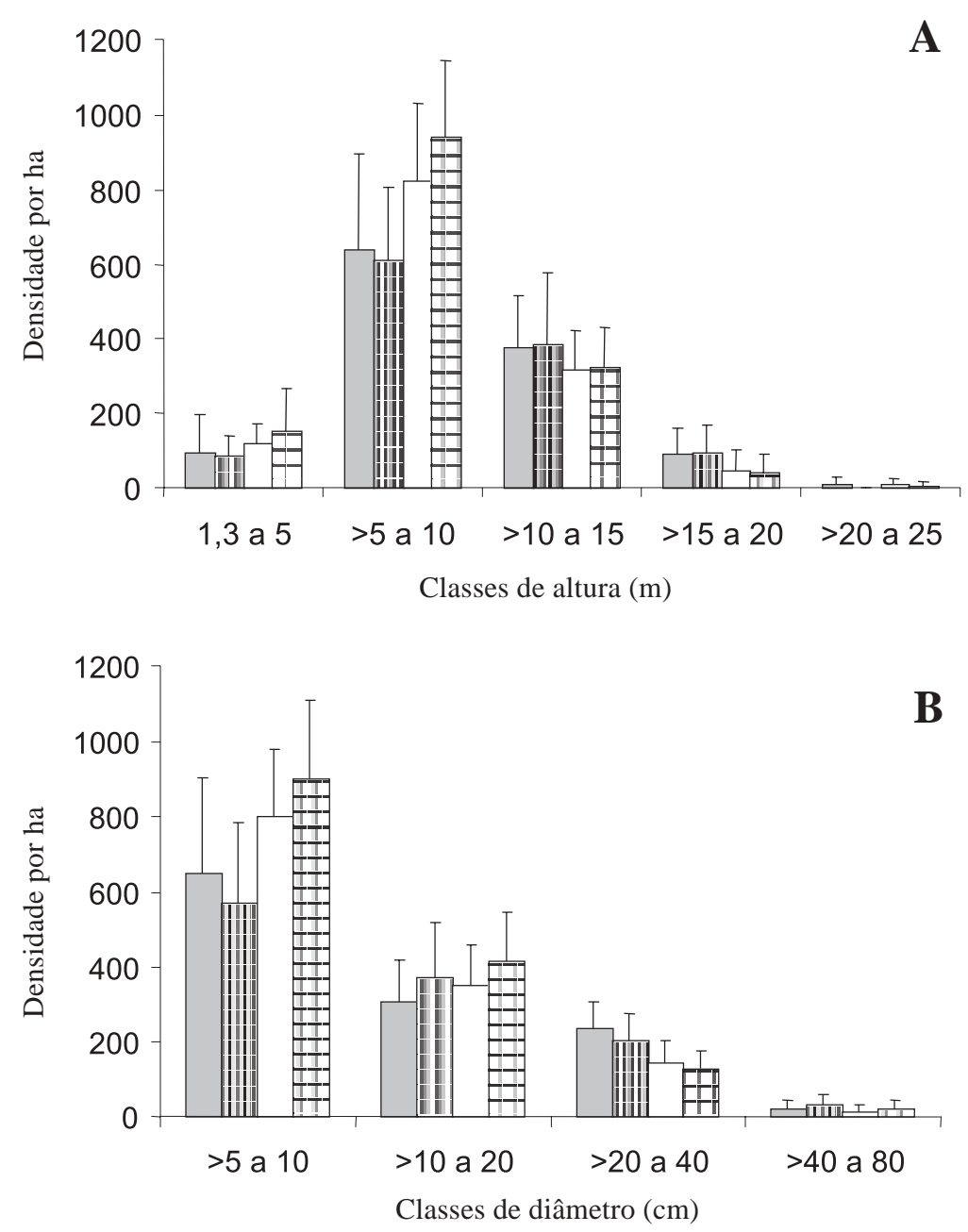

Figura 2. Distribuição da densidade de árvores $\geq 5 \mathrm{~cm}$ de DAP em classes de altura (A) e diâmetro (B) nos quatro setores da Reserva Florestal da UFLA, Lavras, MG. As barras representam a média e as linhas, a média+desvio padrão. $\square$ Interior Alto; $\square$ Borda Alta; $\square$ Interior Baixo; $\boxplus$ Borda Baixa.

As diferenças entre os setores sugerem que os eventos de perturbação e o efeito borda podem ter exercido forte influência nos parâmetros de diversidade de espécies. Atualmente, a biodiversidade tem sido altamente influenciada pelos processos de perda e degradação de habitats naturais e a conseqüente aceleração das taxas de extinção (Hunter 1996; Wilson 1997). Neste contexto, os fragmentos florestais remanescentes sofrem intensamente as conseqüências das perturbações antrópicas, do tamanho reduzido, do efeito de borda (forma), do isolamento e da vizinhança da área (Viana et al. 1997; Viana \& Pinheiro 1998). Assim sendo, o histórico e o grau de modificação destas comunidades podem determinar a extinção de espécies ou a redução drástica de suas populações. De fato, os dois setores do fragmento que sofreram distúrbios mais amenos no passado, Interior Alto e Borda Alta, apresentaram maior diversidade de espécies que seus respectivos parceiros mais intensamente 
perturbados, Interior Baixo e Interior Alto. Por outro lado, as comparações feitas com ênfase na dicotomia entre Interior e Borda sugeriram incremento em diversidade a favor das Bordas. Áreas de bordas podem, muitas vezes, apresentar maior diversidade que o interior dos fragmentos porque podem conservar espécies típicas do interior da floresta às quais são acrescentadas novas espécies exigentes de luz, favorecidas pela maior luminosidade da borda (Williams-Linera 1990b; Viana \& Tabanez 1996; Tabanez et al. 1997; Engel et al. 1998).

Composição das guildas - A distribuição da frequiência de indivíduos arbóreos nas guildas de regeneração não foi independente dos setores da floresta (Tab. 2), sugerindo que o passado de distúrbios e a posição relativa às bordas pode ter interferido na proporção de espécies pioneiras, clímax exigentes de luz e clímax tolerantes à sombra. Árvores de espécies tolerantes à sombra foram mais freqüentes que o esperado nos dois setores Altos, e menos freqüentes que o esperado nos dois setores Baixos. O oposto ocorreu com as árvores de espécies exigentes de luz, mais freqüentes que o esperado nos setores Baixos e menos freqüentes que o esperado nos setores Altos. Já as árvores de espécies pioneiras foram mais abundantes que o esperado nos dois setores de Borda e menos abundantes que o esperado nos dois setores de Interior. Estes resultados sugerem que os setores Altos da mata possuem maior número de espécies características de floresta madura, que podem se desenvolver em condições de maior sombreamento e competição, principalmente no setor Interior Alto. Por outro lado, as proporções significativamente maiores de árvores de espécies exigentes de luz nos dois setores Baixos reforçam as características de fases mais iniciais de regeneração. Quanto às árvores de espécies pioneiras, seu estabelecimento parece ter sido mais favorecido pela maior intensidade luminosa das bordas, conforme já observado por outros autores em outras bordas de florestas tropicais (Williams-Linera 1990b; Williams-Linera et al.
1998; Kellman et al. 1996). A ocorrência das espécies pioneiras no Interior, ainda que em frequiência bem mais baixa, provavelmente se vincula às clareiras que permitem o estabelecimento destas espécies e a manutenção de suas populações (Denslow 1980; Schupp et al. 1989; Tabarelli \& Mantovani 1999a). Contudo, a área de clareiras no interior da floresta é muito inferior à das bordas do fragmento.

A regeneração de uma floresta caminha, geralmente, em direção à maior complexidade de formas, sendo influenciada diretamente pelo tempo decorrente desde a perturbação e pelo tipo de distúrbio (Kageyama et al. 1989; Rondon-Neto et al.2000). Desta forma, esperase que a estratificação da floresta se torne mais complexa com a contribuição crescente de espécies de maior porte para o perfil da floresta. De fato, houve diferenças significativas entre os setores do fragmento na proporção de árvores de espécies de diferentes guildas de estratificação (Tab. 2), sugerindo que a frequiência de espécies próprias de diferentes estratos da floresta varia entre os ambientes estudados. As diferenças mais marcantes concentram-se nos dois setores de Interior, ao passo que não foram verificados contrastes significativos para os setores de Borda. No Interior Alto, a frequiência de árvores de espécies de grande porte (na maturidade) foi maior que o esperado e as frequiências das de médio e pequeno porte (na maturidade) foram menores que o esperado, correspondendo ao perfil de uma fase mais avançada de regeneração. No Interior Baixo foi observado o padrão inverso, confirmando as características esperadas para estádios menos desenvolvidos do processo de regeneração. Também foram encontradas diferenças significativas entre os setores nas proporções de árvores de espécies de diferentes guildas de dispersão, embora isto tenha se verificado apenas para as categorias anemocórica e autocórica (Tab. 2). As espécies zoocóricas perfizeram a grande maioria das árvores de todos os setores, mas não diferiram 
Tabela 2. Tabelas de contingência com as frequiências observadas e esperadas (entre parênteses) de indivíduos arbóreos por guilda de regeneração, estratificação e dispersão nos quatro setores da Reserva Florestal da UFLA, Lavras, MG. Os resultados dos testes de qui-quadrado são fornecidos para cada tabela como um todo e para suas linhas e colunas, em separado.

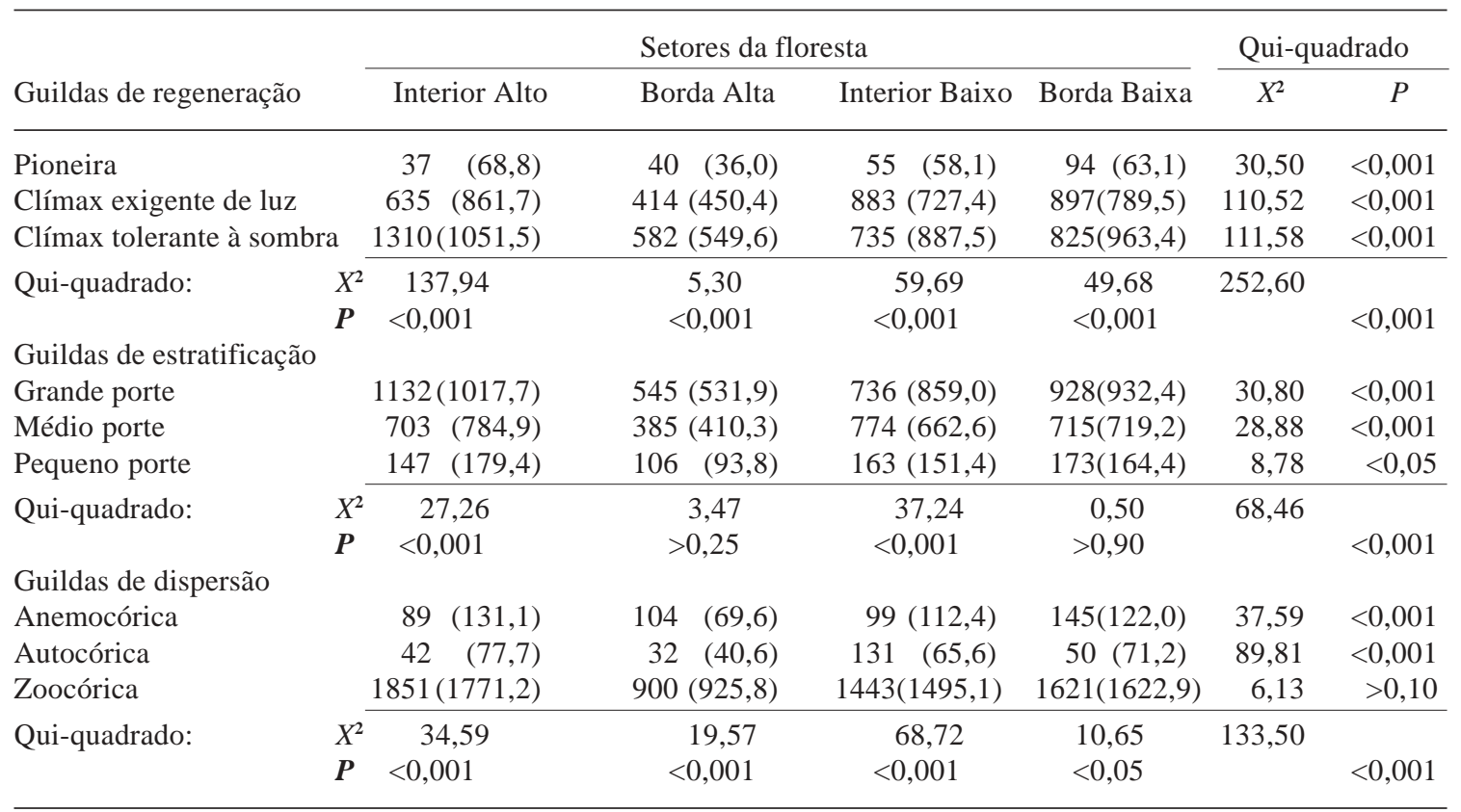

entre eles em suas proporções. As espécies anemocóricas foram mais freqüentes que o esperado nos setores de Borda e menos freqüentes que o esperado nos de Interior. Este achado demonstra a forte influência da maior da exposição das bordas aos ventos, que podem deixar no local maior proporção de diáporos destas espécies que no interior da floresta (Williams-Linera 1990a; 1990b). As espécies autocóricas, bem menos numerosas, foram mais freqüentes que o esperado apenas no Interior Baixo, provavelmente devido à concentração da espécie Maprounea guianensis neste setor.

Relação entre guildas - A relação entre as guildas de regeneração, de dispersão e de tamanho pode ser resultado de mecanismos ecológicos, que durante a regeneração implicam em mudanças direcionais na composição das plantas (Tabarelli \& Mantovani 1999b). A zoocoria predominou em todas as guildas de regeneração, como ocorre na maioria das florestas úmidas tropicais (Piña-Rodrigues et al. 1990). Contudo, as proporções de espécies anemo-autocóricas (dispersão primária abiótica) e zoocóricas variaram significativamente em relação às guildas de regeneração (Tab. 3). As diferenças significativas foram verificadas entre as espécies clímax tolerantes à sombra, para as quais a zoocoria foi mais freqüente e a dispersão abiótica menos freqüente que o esperado. As espécies anemo-autocóricas são mais numerosas que o esperado entre as clímax exigentes de luz e pioneiras e menos numerosas entre as clímax tolerantes à sombra.

As proporções de espécies das guildas de regeneração não variaram significativamente entre as guildas de estratificação, mas houve variações significativas nas proporções das guildas de dispersão entre as guildas de estratificação. $\mathrm{O}$ número de espécies anemoautocóricas foi maior que o esperado nas 
Tabela 3. Tabelas de contingência cruzando a distribuição das freqüências observadas e esperadas (entre parênteses) das espécies arbóreas registradas na Reserva Florestal da UFLA, Lavras, MG, entre as guildas de regeneração, estratificação e dispersão. Os resultados dos testes de qui-quadrado são fornecidos para cada tabela como um todo e para suas linhas e colunas, em separado. Dispersão primária abiótica: anemo+autocórica; sub-bosque: médio+pequeno porte; dossel: grande porte.

\begin{tabular}{|c|c|c|c|c|}
\hline \multirow[b]{2}{*}{ Guildas de regeneração } & \multicolumn{2}{|c|}{ Guildas de dispersão } & \multicolumn{2}{|c|}{ Qui-quadrado } \\
\hline & Abiótica & Zoocórica & $X^{2}$ & $P$ \\
\hline Pioneira & $5(3,6)$ & $9(10,4)$ & 0,77 & $>0,25$ \\
\hline Clímax exigente de luz & $28(21,9)$ & $58(64,1)$ & 2,26 & $>0,10$ \\
\hline Clímax tolerante à sombra & $6(13,5)$ & $47(39,5)$ & 5,60 & $<0,025$ \\
\hline Qui-quadrado: & $X^{2}=6,43 ; P<0,025$ & $X^{2}=2,20 ; P>0,10$ & 8,63 & $<0,025$ \\
\hline \multicolumn{5}{|l|}{ Guildas de estratificação } \\
\hline Guildas de regeneração & Dossel & Sub-bosque & & \\
\hline Pioneira & $5(5,9)$ & $9(8,1)$ & 0,22 & $>0,50$ \\
\hline Clímax exigente de luz & $42(36,0)$ & $44(50,0)$ & 1,74 & $>0,10$ \\
\hline Clímax tolerante à sombra & $17(22,2)$ & $36(30,8)$ & 2,07 & $>0,10$ \\
\hline Qui-quadrado: & $X^{2}=2,34 ; P>0,10$ & $X^{2}=1,68 ; P>0,10$ & 4,02 & $>0,10$ \\
\hline \multicolumn{5}{|l|}{ Guildas de dispersão } \\
\hline Guildas de estratificação & Abiótica & Zoocórica & & \\
\hline Dossel & $25(16,3)$ & $39(47,7)$ & 6,21 & $<0,025$ \\
\hline Sub-bosque & $14(22,7)$ & $75(66,3)$ & 4,64 & $<0,05$ \\
\hline Qui-quadrado: & $X^{2}=7,95 ; P<0,005$ & $X^{2}=2,72 ; P>0,10$ & 10,67 & $<0,005$ \\
\hline
\end{tabular}

espécies do dossel (grande porte) e menor que o esperado entre as de sub-bosque (médio a pequeno porte). Este resultado é justificado pela própria eficiência do mecanismo de dispersão através do vento ou lançamento da própria planta, que aumenta com a altura da árvore e é dificultado no interior da floresta. Mecanismos de dispersão pelo vento ou pela gravidade dependem, além da morfologia das sementes, da altura e/ou posição da árvore dispersora (Pires-O'Brien \& O'Brien 1995). Desta forma, a ocorrência de uma proporção de espécies com dispersão primária abiótica entre as de dossel é esperada devido à própria eficiência do processo de disseminação. Entre as espécies de subbosque, o número de espécies zoocóricas foi maior que o esperado, sugerindo maior eficiência da dispersão por animais no interior da floresta, onde é favorecido o acesso de primatas e outros pequenos mamíferos aos frutos.

Concluindo, os resultados encontrados neste estudo reforçam a consistência da interpretação das variações fisionômicas do fragmento florestal da Reserva Florestal da UFLA como resultado do efeito borda e das diferenças de intensidade de perturbação sofridas no passado. O presente estudo explorou novos aspectos das variações da comunidade arbórea e forneceu evidências de que uma análise integrada de certas caracterísicas, como a densidade, área basal, estrutura de tamanhos, diversidade e composição de guildas de regeneração, estratificação e dispersão podem servir como bons avaliadores da severidade da perturbação sofrida no passado por estas florestas, da fase regenerativa atual bem como do impacto do efeito borda. 
Apêndice. Espécies arbóreas da Reserva Florestal da UFLA, Lavras, MG, seguidas de suas respectivas guildas de regeneração (Reg.), estratificação (Estr.) e dispersão (Disp.) e número de indivíduos registrados nos quatro setores da floresta: Interior Alto (Int.Al.), Borda Alta (Bor.Al.), Interior Baixo (Int.Bx.) e Borda Baixa (Bor.Bx.). Guildas: Pio = pioneira; Luz = clímax exigente de luz; Som = clímax tolerante a sombra; Grd = grande; Méd = médio, Peq = pequeno; Ane $=$ anemocórica; Aut = autocórica; Zoo = zoocórica. Espécies organizadas por famílias e em ordem alfabética.

\begin{tabular}{llllll}
\hline \multirow{2}{*}{ Famílias e espécies } & \multicolumn{3}{c}{ Guildas } & & N. de indivíduos nos setores \\
\cline { 5 - 6 } & Reg. & Estr. & Disp. & & Int.Al. Bor.Al. Int.Bx. Bor.Bx. \\
\hline
\end{tabular}

ANACARDIACEAE

Astronium graveolens Jacquin

Tapirira guianensis Aublet

T. obtusa (Benth.) Mitchell

ANNONACEAE

Annona cacans Warm.

Duguetia lanceolata A.St.-Hil.

Guatteria nigrescens Mart.

Rollinia laurifolia Schltdl.

Som

Pio

Grd

Luz

$R$. sericea (R.E.Fries) R.E.Fries

Xylopia brasiliensis Sprengel

APOCYNACEAE

Aspidosperma spruceanum Benth.

AQUIFOLIACEAE

Ilex cerasifolia Reissek

ARALIACEAE

Dendropanax cuneatus (DC.) Decne \& Planchon Som

Schefflera longepetiolata (E.Marchal) D.Frodin Som

ARECACEAE

Geonoma schottiana Mart.

Syagrus flexuosa (Mart.) Becc.

S. romanzoffiana (Cham.) Glassman

ASTERACEAE

Piptocarpha macropoda Baker

BIGNONIACEAE

Cybistax antisyphillitica Mart.

Jacaranda macrantha Cham.

Jacaranda puberula Cham.

Tabebuia serratifolia (Vahl) Nichols

Zeyheria tuberculosa (Vell.) Bureau

BORAGINACEAE

Cordia sellowiana Cham.

BURSERACEAE

Protium spruceanum (Benth.) Engler

P. widgrenii Engler

CANELLACEAE

Cinnamodendron dinisii Schwacke

CECROPIACEAE

Cecropia pachystachya Trécul

CELASTRACEAE

Salacia elliptica (Mart.) G.Don.

CHRYSOBALANACEAE

Hirtella hebeclada Moric.

CLETHRACEAE

Clethra scabra Pers.

$\begin{array}{lcccccc}\text { Luz } & \text { Grd } & \text { Zoo } & 2 & - & - & 3 \\ \text { Som } & \text { Grd } & \text { Zoo } & 36 & 20 & 2 & 6 \\ \text { Som } & \text { Méd } & \text { Zoo } & 6 & 3 & 7 & 9 \\ \text { Luz } & \text { Grd } & \text { Zoo } & 7 & 8 & 1 & 14 \\ \text { Luz } & \text { Grd } & \text { Zoo } & - & 1 & 1 & 5 \\ \text { Som } & \text { Grd } & \text { Zoo } & 126 & 30 & 149 & 199\end{array}$

$\begin{array}{lllllll}\text { Luz } & \text { Grd } & \text { Ane } & 1 & 1 & - & 1\end{array}$

Som Méd Zoo $\quad 2 \quad \begin{array}{lllll} & 2 & 4 & 6\end{array}$

Méd Zoo

Méd Zoo

Som

Luz

Luz

Peq Zoo

Peq Zoo

Grd Zoo

Pio Méd Ane

Luz

Luz

Peq Ane

Luz

Grd

Grd

Ane

Som

Grd

Ane

Luz

Grd

Ane

Ane

Luz Grd Zoo

27

Som

Grd

Zoo

Luz

Méd

Zoo

$-$

Som

Grd

Zoo

Pio Méd Zoo

Som

Peq

Zoo

8

3

5

3

Som

Mé

Zoo

32

11

9

5

Luz Méd Ane
2 
Apêndice (continuação)

\begin{tabular}{|c|c|c|c|c|c|c|c|}
\hline \multirow[b]{2}{*}{ Famílias e espécies } & \multicolumn{3}{|c|}{ Guildas } & \multicolumn{4}{|c|}{ N. de indivíduos nos setores } \\
\hline & Reg. & Estr. & Disp. & Int.Al. & Bor.Al. & Int.Bx. & Bor.Bx. \\
\hline \multicolumn{8}{|l|}{ CLUSIACEAE } \\
\hline Garcinia gardneriana (Planchon \& Triana) Zappi & Som & Méd & Zoo & 9 & - & - & - \\
\hline Vismia brasiliensis Choisy & Luz & Méd & Zoo & 4 & 3 & 1 & 10 \\
\hline \multicolumn{8}{|l|}{ CONNARACEAE } \\
\hline $\begin{array}{l}\text { Connarus regnellii G.Schelenb. } \\
\text { CUNONIACEAE }\end{array}$ & \multicolumn{6}{|c|}{ CUNONIACEAE } & - \\
\hline $\begin{array}{l}\text { Lamanonia ternata Vell. } \\
\text { ELAEOCARPACEAE }\end{array}$ & Luz & Méd & Ane & 5 & 2 & 6 & 23 \\
\hline $\begin{array}{l}\text { Sloanea monosperma Vell. } \\
\text { ERYTHROXYLACEAE }\end{array}$ & Som & Grd & Zoo & 1 & - & - & - \\
\hline $\begin{array}{l}\text { Erythroxylum pelleterianum A.St.-Hil. } \\
\text { EUPHORBIACEAE }\end{array}$ & Som & Peq & Zoo & 2 & 1 & 1 & 6 \\
\hline Actinostemon concolor (Sprengel) Müll.Arg. & Som & Méd & Aut & 1 & - & - & - \\
\hline Alchornea triplinervea (Sprengel) Müll.Arg. & Luz & Grd & Zoo & - & - & 2 & - \\
\hline Croton floribundus Sprengel & Pio & Grd & Aut & 8 & 8 & - & 7 \\
\hline Maprounea guianensis Aublet & Luz & Méd & Aut & 20 & 18 & 131 & 43 \\
\hline Pera glabrata (Schott) Poepp. & Luz & Grd & Zoo & 5 & 9 & 3 & 10 \\
\hline \multicolumn{8}{|l|}{ FABACEAE } \\
\hline Albizia polycephala (Benth.) Killip & Luz & Grd & Ane & 5 & 1 & - & 1 \\
\hline Anadenanthera colubrina (Vell.) Brenan & Pio & Grd & Ane & - & - & - & 2 \\
\hline Bowdichia virgilioides Kunth & Luz & Grd & Ane & 3 & 1 & 31 & 11 \\
\hline Cassia ferruginea (Schrad.) Schrad. & Luz & Grd & Zoo & - & 1 & - & - \\
\hline Copaifera langsdorffii Desf. & Som & Grd & Zoo & 166 & 79 & 157 & 143 \\
\hline Dalbergia nigra (Vell.) Fr.Allem. & Luz & Grd & Ane & - & - & 1 & 1 \\
\hline D. villosa (Benth.) Benth. & Luz & Grd & Ane & 1 & 2 & - & - \\
\hline Hymenaea courbaril $\mathrm{L}$. & Luz & Grd & Zoo & 34 & 15 & 1 & 4 \\
\hline Inga striata Benth. & Luz & Grd & Zoo & - & - & - & 1 \\
\hline Leucochloron incuriale (Vell.) Barneby \& Grimes & Luz & Grd & Ane & 1 & 2 & - & 2 \\
\hline Machaerium amplum Benth. & Luz & Méd & Ane & - & 1 & 1 & 2 \\
\hline M. brasiliense Vogel & Luz & Grd & Ane & - & - & - & 2 \\
\hline M. hirtum (Vell.) Stellfeld & Luz & Grd & Ane & - & 1 & - & - \\
\hline M. nictitans (Vell.) Benth. & Luz & Grd & Ane & 13 & 10 & 3 & 4 \\
\hline M. stipitatum (DC.) Vogel & Luz & Grd & Ane & - & - & 1 & - \\
\hline M. triste Vogel & Luz & Méd & Ane & 1 & - & - & 2 \\
\hline M. villosum Vogel & Luz & Grd & Ane & 4 & 3 & 2 & 11 \\
\hline Ormosia arborea (Vell.) Harms & Luz & Grd & Zoo & 1 & - & - & - \\
\hline Piptadenia gonoacantha (Mart.) Macbr. & Pio & Grd & Ane & - & 6 & - & 2 \\
\hline Platycyamus regnellii Benth. & Luz & Grd & Ane & 4 & 3 & - & - \\
\hline Platypodium elegans Vogel & Luz & Grd & Ane & 1 & 4 & - & 4 \\
\hline Pseudopiptadenia leptostachya (Benth.) Rausch. & Som & Méd & Ane & 2 & - & - & - \\
\hline Sclerolobium rugosum Mart. & Luz & Grd & Ane & 13 & 14 & 33 & 30 \\
\hline Senna macranthera (Vell.) Irwin \& Barneby & Pio & Méd & Zoo & - & 1 & - & 2 \\
\hline \multicolumn{8}{|l|}{ FLACOURTIACEAE } \\
\hline Casearia arborea (L.C.Rich.) Urban & Luz & Grd & Zoo & 92 & 72 & 100 & 189 \\
\hline C. decandra Jacquin & Som & Méd & Zoo & 1 & 2 & - & 4 \\
\hline C. lasiophylla Eichler & Luz & Peq & Zoo & 1 & - & - & 2 \\
\hline C. obliqua Sprengel & Luz & Grd & Zoo & - & 1 & - & 2 \\
\hline C. sylvestris Swartz & Pio & Méd & Zoo & 2 & 4 & - & 32 \\
\hline \multicolumn{8}{|l|}{ LAURACEAE } \\
\hline Cryptocarya aschersoniana $\mathrm{Mez}$ & Som & Grd & Zoo & 87 & 25 & 13 & $\begin{array}{l}16 \\
\text { continuc }\end{array}$ \\
\hline
\end{tabular}


Apêndice (continuação)

\begin{tabular}{|c|c|c|c|c|c|c|c|}
\hline \multirow[b]{2}{*}{ Famílias e espécies } & \multicolumn{3}{|c|}{ Guildas } & \multicolumn{4}{|c|}{ N. de indivíduos nos setores } \\
\hline & Reg. & Estr. & Disp. & Int.A1. & Bor.Al. & Int.Bx. & Bor.Bx. \\
\hline \multicolumn{8}{|l|}{ LAURACEAE } \\
\hline Nectandra grandiflora Nees & Som & Grd & Zoo & 2 & 2 & - & - \\
\hline N. oppositifolia Nees & Som & Grd & Zoo & 5 & 3 & - & 4 \\
\hline Ocotea corymbosa (Meisner) Mez & Luz & Grd & Zoo & 74 & 38 & 51 & 48 \\
\hline O. diospyrifolia (Meisner) Mez & Luz & Grd & Zoo & 2 & 1 & - & - \\
\hline O. laxa (Nees) Mez & Som & Peq & Zoo & 2 & 11 & 1 & 8 \\
\hline O. odorifera (Vell.) Rohwer & Som & Grd & Zoo & 262 & 59 & 26 & 13 \\
\hline Persea pyrifolia Nees \& Mart. & Luz & Grd & Zoo & 10 & 8 & 33 & 23 \\
\hline \multicolumn{8}{|l|}{ MALPIGHIACEAE } \\
\hline Byrsonima laxiflora Griseb. & Luz & Grd & Zoo & 19 & - & 7 & 12 \\
\hline Heteropterys byrsonimifolia A.Juss. & Pio & Méd & Ane & 7 & 5 & 11 & 29 \\
\hline \multicolumn{8}{|l|}{ MALVACEAE } \\
\hline Guazuma ulmifolia Lam. & Pio & Grd & Zoo & 3 & - & - & - \\
\hline Luehea grandiflora Mart. \& Zucc. & Luz & Grd & Ane & 1 & 10 & - & 3 \\
\hline \multicolumn{8}{|l|}{ MELASTOMATACEAE } \\
\hline Miconia albicans Triana & Pio & Peq & Zoo & 1 & - & - & 1 \\
\hline M. argyrophylla DC. & Luz & Méd & Zoo & 89 & 30 & 96 & 87 \\
\hline M. chartacea Triana & Luz & Méd & Zoo & 7 & - & 41 & 6 \\
\hline M. cinnamomifolia (DC.) Naudin & Pio & Grd & Zoo & 1 & 1 & - & 1 \\
\hline M. pepericarpa DC. & Pio & Peq & Zoo & 6 & - & 39 & 10 \\
\hline M. trianae Cogn. & Luz & Méd & Zoo & 7 & 1 & 83 & 13 \\
\hline M. tristis Sprengel & Luz & Méd & Zoo & - & - & 1 & - \\
\hline Tibouchina stenocarpa (DC.) Cogn. & Luz & Méd & Ane & 1 & - & - & - \\
\hline \multicolumn{8}{|l|}{ MELIACEAE } \\
\hline Cabralea canjerana (Vell.) Mart. & Som & Grd & Zoo & 1 & 1 & - & 1 \\
\hline Cedrela fissilis Vell. & Luz & Grd & Ane & - & 1 & - & - \\
\hline Guarea macrophylla Vahl. & Som & Méd & Zoo & - & - & - & 1 \\
\hline Trichilia emarginata (Turcz.) C.DC. & Som & Méd & Zoo & 161 & 28 & 16 & 44 \\
\hline T. pallida Swartz & Som & Méd & Zoo & 1 & 1 & - & 1 \\
\hline \multicolumn{8}{|l|}{ MEMECYLACEAE } \\
\hline Mouriri glazioviana Cogn. & Luz & Méd & Zoo & 4 & - & 11 & 2 \\
\hline \multicolumn{8}{|l|}{ MONIMIACEAE } \\
\hline Mollinedia argyrogyna Perkins & Som & Méd & Zoo & 1 & - & - & - \\
\hline M. widgrenii A.DC. & Som & Méd & Zoo & 8 & 28 & - & 1 \\
\hline \multicolumn{8}{|l|}{ MORACEAE } \\
\hline Ficus pertusa L.f. & Som & Grd & Zoo & 3 & 2 & - & 6 \\
\hline Sorocea bonplandii (Baillon) W.Burger & Som & Méd & Zoo & 1 & 1 & - & - \\
\hline \multicolumn{8}{|l|}{ MYRSINACEAE } \\
\hline Myrsine gardneriana A.DC. & Luz & Méd & Zoo & 6 & 2 & 32 & 10 \\
\hline M. umbellata Mart. & Luz & Méd & Zoo & 3 & 3 & 5 & 1 \\
\hline \multicolumn{8}{|l|}{ MYRTACEAE } \\
\hline Blepharocalyx salicifolius (Kunth) O.Berg & Luz & Méd & Zoo & 6 & 3 & 21 & 17 \\
\hline Calycorectes acutatus (Miq.) Toledo & Som & Méd & Zoo & 11 & 1 & - & - \\
\hline Calyptranthes brasiliensis Sprengel & Som & Méd & Zoo & 2 & - & 6 & - \\
\hline C. clusiifolia (Miq.) O.Berg & Som & Méd & Zoo & 23 & 12 & 14 & 24 \\
\hline Eugenia florida DC. & Som & Méd & Zoo & 1 & 1 & - & - \\
\hline E. handroana D.Legrand & Som & Méd & Zoo & 1 & - & - & 1 \\
\hline E. hyemalis Cambess. & Luz & Méd & Zoo & 5 & 1 & 4 & 14 \\
\hline Gomidesia affinis (Cambess.) D.Legrand & Luz & Méd & Zoo & - & 1 & - & 1 \\
\hline
\end{tabular}


Apêndice (continuação)

\begin{tabular}{|c|c|c|c|c|c|c|c|}
\hline \multirow[b]{2}{*}{ Famílias e espécies } & \multicolumn{3}{|c|}{ Guildas } & \multicolumn{4}{|c|}{ N. de indivíduos nos setores } \\
\hline & Reg. & Estr. & Disp. & Int.Al. & Bor.Al. & Int.Bx. & Bor.Bx. \\
\hline \multicolumn{8}{|l|}{ MYRTACEAE } \\
\hline Gomidesia eriocalyx (DC.) O.Berg & Luz & Méd & Zoo & - & - & 1 & 1 \\
\hline Marlierea racemosa (Vell.) Kiaersk. & Luz & Méd & Zoo & 4 & 2 & - & 2 \\
\hline Myrcia fallax (Rich.) DC. & Luz & Méd & Zoo & 28 & 5 & 44 & 83 \\
\hline M. rostrata $\mathrm{DC}$ & Luz & Méd & Zoo & - & - & - & 2 \\
\hline M. tomentosa (Aublet) DC. & Luz & Méd & Zoo & - & 2 & - & - \\
\hline M. venulosa $\mathrm{DC}$. & Luz & Méd & Zoo & 2 & - & 10 & 14 \\
\hline Myrciaria floribunda (West) O.Berg & Som & Méd & Zoo & - & 1 & - & - \\
\hline Pimenta pseudocaryophyllus (Gomes) Landrum & Luz & Méd & Zoo & 2 & 4 & - & - \\
\hline Psidium cattleyanum Sabine & Luz & Méd & Zoo & - & 1 & - & - \\
\hline P. rufum Mart. & Luz & Méd & Zoo & 2 & 3 & - & - \\
\hline Siphoneugena densiflora O.Berg & Som & Méd & Zoo & 10 & 4 & 11 & 1 \\
\hline S. kuhlmannii Mattos & Som & Méd & Zoo & 18 & 4 & 25 & 10 \\
\hline \multicolumn{8}{|l|}{ NYCTAGINACEAE } \\
\hline Guapira graciliflora (Schmidt) Lundell & Som & Méd & Zoo & 1 & - & - & 1 \\
\hline G. opposita (Vell.) Reitz & Som & Méd & Zoo & 1 & 1 & - & - \\
\hline \multicolumn{8}{|l|}{ OCHNACEAE } \\
\hline $\begin{array}{l}\text { Ouratea semiserrata (Mart. \& Nees) Engler } \\
\text { OLACACEAE }\end{array}$ & Som & Peq & Zoo & 1 & 1 & 2 & - \\
\hline Heisteria silvianii Schwacke & Som & Méd & Zoo & 4 & 2 & - & 1 \\
\hline \multicolumn{8}{|l|}{ POLYGONACEAE } \\
\hline Coccoloba warmingii Meisner & Luz & Méd & Zoo & 2 & - & - & - \\
\hline \multicolumn{8}{|l|}{ RHAMNACEAE } \\
\hline Colubrina glandulosa Perkins & Luz & Grd & Zoo & - & 2 & - & - \\
\hline \multicolumn{8}{|l|}{ RUBIACEAE } \\
\hline Alibertia concolor (Cham.) K.Schum. & Luz & Méd & Zoo & 1 & - & - & 4 \\
\hline Amaioua guianensis Aublet & Som & Méd & Zoo & 97 & 37 & 160 & 154 \\
\hline Faramea cyanea Müll.Arg. & Som & Méd & Zoo & 5 & 1 & 4 & - \\
\hline Guettarda uruguensis Cham. \& Schltdl. & Luz & Méd & Zoo & - & 1 & - & - \\
\hline Ixora warmingii Müll.Arg. & Som & Méd & Zoo & 31 & 83 & - & 7 \\
\hline Psychotria sessilis (Vell.) Müll.Arg. & Pio & Méd & Zoo & 1 & - & - & 1 \\
\hline Rudgea viburnoides (Cham.) Benth. & Luz & Peq & Zoo & - & - & - & 4 \\
\hline \multicolumn{8}{|l|}{ RUTACEAE } \\
\hline Galipea jasminiflora (A.St.-Hil.) Engler & Som & Méd & Aut & 8 & 3 & - & - \\
\hline Metrodorea stipularis Mart. & Som & Grd & Aut & 5 & 3 & - & - \\
\hline Zanthoxylum caribaeum Lam. & Luz & Grd & Zoo & - & 1 & - & - \\
\hline Z. fagara (L.) Sargent & Luz & Méd & Zoo & - & - & 2 & - \\
\hline Z. rhoifolium Lam. & Luz & Grd & Zoo & - & 3 & - & 1 \\
\hline \multicolumn{8}{|l|}{ SAPINDACEAE } \\
\hline Cupania vernalis Cambess. & Luz & Grd & Zoo & - & 1 & - & - \\
\hline Diatenopteryx sorbifolia Radlk. & Luz & Méd & Ane & 8 & 10 & - & - \\
\hline \multicolumn{8}{|l|}{ SAPOTACEAE } \\
\hline $\begin{array}{l}\text { Chrysophyllum gonocarpum } \\
\text { (Mart. \& Eichler) Engler }\end{array}$ & Luz & Méd & Zoo & - & 1 & - & - \\
\hline \multicolumn{8}{|l|}{ SIPARUNACEAE } \\
\hline Siparuna guianensis Aublet & Som & Peq & Zoo & 123 & 90 & 103 & 136 \\
\hline \multicolumn{8}{|l|}{ SOLANACEAE } \\
\hline Solanum pseudoquina A.St.-Hil. & Luz & Méd & Zoo & - & 1 & - & - \\
\hline \multicolumn{8}{|l|}{ STYRACACEAE } \\
\hline Styrax camporus Pohl & Luz & Méd & Zoo & - & - & 1 & $\begin{array}{c}2 \\
\text { continu }\end{array}$ \\
\hline
\end{tabular}


Apêndice (continuação)

\begin{tabular}{|c|c|c|c|c|c|c|c|}
\hline \multirow[b]{2}{*}{ Famílias e espécies } & \multicolumn{3}{|c|}{ Guildas } & \multicolumn{4}{|c|}{ N. de indivíduos nos setores } \\
\hline & Reg. & Estr. & Disp. & Int.Al. & Bor.Al. & Int.Bx. & Bor.Bx. \\
\hline \multicolumn{8}{|l|}{ STYRACACEAE } \\
\hline Styrax latifolius Pohl & Som & Grd & Zoo & 29 & 15 & 7 & 7 \\
\hline \multicolumn{8}{|l|}{ THEACEAE } \\
\hline Ternstroemia brasiliensis Cambess. & Luz & Méd & Zoo & - & - & - & 1 \\
\hline THYMELAEACEAE & & & & & & & \\
\hline Daphnopsis fasciculata (Meisner) Nevling & Luz & Méd & Zoo & 3 & 2 & 1 & - \\
\hline \multicolumn{8}{|l|}{ VERBENACEAE } \\
\hline Vitex cymosa Bert. & Luz & Grd & Zoo & 5 & 6 & - & 3 \\
\hline V. polygama Cham. & Luz & Méd & Zoo & 4 & 3 & - & - \\
\hline \multicolumn{8}{|l|}{ VOCHYSIACEAE } \\
\hline Qualea grandiflora Mart. & Luz & Méd & Zoo & - & 1 & 3 & 2 \\
\hline Vochysia tucanorum Mart. & Luz & Méd & Ane & - & 2 & - & - \\
\hline
\end{tabular}

\section{Referências bibliográficas}

Barroso, G. M.; Morim, M. P.; Peixoto, A. L. \& Ichaso, C. L. F. 1999. Frutos e sementes: morfologia aplicada à sistemática de dicotiledôneas. Editora UFV, Viçosa.

Botrel, R. T.; Oliveira-Filho, A. T.; Rodrigues, L. A. \& Curi, N. 2002. Composição florística e estrutura da comunidade arbórea de um fragmento de floresta estacional semidecidual em Ingaí, MG, e a influência de variáveis ambientais na distribuição das espécies. Revista Brasileira de Botânica 25(2): 195-213.

Chagas, R. K.; Oliveira-Filho, A. T.; Van den Berg, E. \& Scolforo, J. R. S. 2001. Dinâmica de populações arbóreas em um fragmento de floresta estacional semidecidual montana em Lavras, Minas Gerais. Revista Árvore 25(1): 39-57.

Clark, D. A. 1994. Plant demography. Pp. 90-105. In: L. A. McDade; K. S. Bawa; H. A. Hespenheide \& G. S. Hartshorn (Eds.). La Selva: ecology and natural history of a neotropical rain forest. University of Chicago Press, Chicago.

Condit, R.; Sukumar, R.; Hubbell, S. P. \& Foster, R. B. 1998. Predicting population trends from size distribution: a direct test in a tropical tree community. The American Naturalist 152(4): 495-509.

Denslow, J. S. 1980. Gap partitioning among tropical rainforest trees. Biotropica 12 (Suppl.): 47-55.

DNMet (Departamento Nacional de Meteorologia). 1992. Normais climatológicas (1961-1990). Ministério da Agricultura, Brasília.
EMBRAPA 1999. Sistema brasileiro de classificação de solos. Empresa Brasileira de Pesquisa Agropecuária, Centro Nacional de Pesquisa de Solos, Rio de Janeiro.

Engel, V. L.; Fonseca, R. C. B. \& Oliveira, R. E. 1998. Ecologia de lianas e o manejo de fragmentos florestais. Série Técnica IPEF 12(32): 43-64.

Gaston, K. J. 1996. What is biodiversity? Pp. 1-9. In: K. J. Gaston (Ed.). Biodiversity: a biology of numbers and differences. Blackwell Science, London.

Hunter, M. L. 1996. Fundamentals of conservation biology. Blackwell Science, Cambridge.

Hutchings, M. J. 1997. The structure of plant populations. Pp. 325-358. In: M. J. Crawley (Ed.). Plant ecology. $2^{\text {nd }}$ edition. Blackwell Science, London.

Kageyama, P. Y.; Castro, C. F. A. \& Carpanezzi, A. A. 1989. Implantação de matas ciliares: estratégias para auxiliar a sucessão secundária. Pp. 136-143. In: Anais do Simpósio Sobre Mata Ciliar. Fundação Cargill, Campinas.

Kapos, V. 1989. Effects of isolation on the water status of forest patches in the Brazilian Amazon. Journal of Tropical Ecology 5(2): 173-185.

Kellman, M.; Tackaberry, R. \& Meave, J. 1996. The consequences of prolonged fragmentation: lessons from tropical gallery forests. Pp. 37-58. In: J. Schelhas \& R. Greenberg (Eds.). Forest patches in tropical landscapes. Island Press, Washington.

Krebs, C. J. 1989. Ecological methodology. Harper and Row, New York.

Laurance, W. F. \& Yensen, E. 1991. Predicting the impacts of edges in fragmented habitats. Biological Conservation 55(1): 77-92. 
Lorenzi, H. 1992. Árvores brasileiras; Manual de identificação e cultivo de plantas arbóreas nativas do Brasil. Vol. 1, Ed. Plantarum, Nova Odessa.

Lorenzi, H. 1992. Árvores brasileiras; Manual de identificação e cultivo de plantas arbóreas nativas do Brasil. Vol. 2, Ed. Plantarum, Nova Odessa.

Lovejoy, T. E. \& Bierregaard, R. O. 1990. Central Amazonian forests and the minimum critical size of ecosystems project. Pp. 60-71. In: A. H. Gentry (Ed.). Four neotropical rainforests. Yale University Press, New Haven.

Lovejoy, T. E.; Bierregaard, R. O.; Rankin, J. M. \& Schubart, H. O. R. 1983. Ecological dynamics of forest fragments. Pp. 377-384. In: S. L. Sutton; T. C. Whitmore \& A. C. Chadwick (Eds.). Tropical rainforest: ecology and management. Blackwell Science, Oxford.

Lovejoy, T. E.; Rankin, J. M.; Bierregaard, R. O.; Brown, K. S.; Emmons, L. H. \& van der Voot, M. 1984. Ecosystem decay of Amazon forest remmants. Pp. 295-325. In: N. H. Nitecki (Ed.). Extinctions. University of Chicago Press, Chicago.

Lovejoy, T. E.; Bierregaard, R. O.; Rylands, A. B.; Malcolm, J. R.; Quintela, C. E.; Harper, L. H.; Brown, K. S.; Powell, A. H.; Powell, G. V. N.; Schubart, H. O. R. \& Hays, M.B. 1986. Edges and other effects of isolation on Amazon forest fragments. Pp. 257-285. In: M. E. Soulé (Ed.). Conservation biology: the science of scarcity and diversity. Sianuer, Sunderland.

Morellato, L. P. C. \& Leitão-Filho, H. F. 1992. Padrões de frutificação e dispersão na Serra do Japi. Pp. 112-141. In: L. P. C. Morellato (Org.). História natural da Serra do Japi: ecologia e preservação de uma área no Sudeste do Brasil. Editora da UNICAMP/FAPESP, Campinas.

Metzger, J. P. 1999. Estrutura da paisagem e fragmentação: análise bibliográfica. Anais da Academia Brasileira de Ciências 71(3I): 445-463.

Murcia, C. 1995. Edge effects in fragmented forests: implications for conservation. Trends in Ecology and Evolution 10(1): 58-62.

Oliveira-Filho, A. T.; Scolforo, J. R. S. \& Mello, J. M. 1994. Composição florística e estrutura comunitária de um remanescente de floresta semidecídua em Lavras, MG. Revista Brasileira de Botânica 17(2): 167-182.

Oliveira-Filho, A. T.; Vilela, E. A.; Carvalho, D. A. \& Gavilanes, M. L. 1995. Estudos florísticos e fitossociológicos em remanescentes de matas ciliares do Alto e Médio Rio Grande. Boletim técnico 11.106-MA/PA-013, Companhia Energética de Minas Gerais (CEMIG), Belo Horizonte.
Oliveira-Filho, A. T.; Mello, J. M. \& Scolforo, J. R. S. 1997. Effects of past disturbance and edges on tree community structure and dynamics within a fragment of tropical semideciduous forest in Southeastern Brazil over a five-year period (1987-1992). Plant Ecology 131(1): 45-66.

Parthasarathy, N. 1999. Tree diversity and distribution in undisturbed and human-impacted sites of tropical wet evergreen forest in southern Western Ghats, India. Biodiversity and Conservation 8(4): 1365-1381.

Pinã-Rodrigues, F. C. M.; Costa, L. G. S. \& Reis, A. 1990. Estratégias de estabelecimento de espécies arbóreas e o manejo de florestas tropicais. Pp. 677684. In: Anais do $6^{\circ}$ Congresso Florestal Brasileiro. Campos de Jordão.

Pires-O'Brien, M. J. \& O'Brien, C. M. 1995. Ecologia e modelamento de florestas tropicais. FCAP, Belém.

Pulz, F. A. 1998. Estudo da dinâmica e a modelagem da estrutura diamétrica de uma floresta semidecídua montana na região de Lavras - MG. Dissertação de Mestrado. Universidade Federal de Lavras, Lavras.

Rondon-Neto, R. M.; Botelho, S. A.; Fontes, M. A. L.; Davide, A. C. \& Faria, J. M. R. 2000. Estrutura e composição florística da comunidade arbustivoarbórea de uma clareira de formação antrópica, em uma floresta estacional semidecídua montana. Cerne 6(2): 79-94.

Schupp, E. W.; Howe, H. F.; Augspurger, C. K. \& Levey, D. J. 1989. Arrival and survival in tropical treefall gaps. Ecology 70(3): 562-564.

Swaine, M. D. \& Whitmore, T. C. 1988. On the definition of ecological species groups in tropical rain forests. Vegetatio 75(1): 81-86.

Tabanez, A. A. J.; Viana, V. M. \& Dias, A. S. 1997. Conseqüências da fragmentação e do efeito de borda sobre a estrutura, diversidade e sustentabilidade de um fragmento de floresta de planalto de Piracicaba, SP. Revista Brasileira de Biologia 57(1): 47-60.

Tabarelli, M. \& Mantovani, W. 1999a. Clareiras naturais e a riqueza de espécies pioneiras em uma floresta Atlântica montana. Revista Brasileira de Biologia 59(2): 251-261.

Tabarelli, M. \& Mantovani, W. 1999b. A regeneração de uma floresta tropical montana após corte e queima (São Paulo-Brasil). Revista Brasileira de Biologia 59(2): 239-250.

Van der Pijl, L. 1982. Principles of dispersal in higher plants. $3^{\text {rd }}$ ed. Springer-Verlag, Berlin. 
Uhl, C. \& Murphy, P. G. 1981. Composition, structure, and regeneration of a "tierra firme" forest in the Amazon Basin of Venezuela. Tropical Ecology 22(2): 219-237.

Viana, V. M. \& Pinheiro, L. A. F. V. 1998. Conservação da biodiversidade em fragmentos florestais. Série Técnica IPEF 12(32): 25-42.

Viana, V. M. \& Tabanez, A. A. J. 1996. Biology and conservation of forest fragments in the Brazilian Atlantic Moist Forest. Pp. 151-167. In: J. Schelhas \& R. Greenberg (Eds.). Forest patches in tropical landscapes. Island Press, Washington.

Viana, V. M.; Tabanez, A. A. J. \& Batista, J. L. 1997. Restoration and management of fragmented landscapes. Pp. 347-365. In: W. F. Laurance \& R. O. Bierregaard (Eds.). Tropical forest remnants: ecology, management, and conservation of fragmented communities. University of Chicago Press, Chicago.
Whitmore, T. C. 1990. An introdution to the tropical rain forests. Clarendon Press, Oxford.

Williams-Linera, G. 1990a. Vegetation structure and environmental conditions of forest edges in Panama. Journal of Ecology 78(2): 356-373.

Williams-Linera, G. 1990b. Origin and early development of forest edge vegetation in Panama. Biotropica 22(3): 235-241.

Williams-Linera, G.; Domínguez-Gastelú, V. \& GarcíaZurita, M. E. 1998. Microenvironment and floristics of different edges in a fragmented tropical rainforest. Conservation Biology 12(5): 1091-1102.

Wilson, E. O. 1997. A situação atual da diversidade biológica. Pp. 3-24. In: E. O. Wilson \& F. M. Peter (Eds.). Biodiversidade. Nova Fronteira, Rio de Janeiro.

Zar, J. H. 1996. Biostatistical analysis. $3^{\text {rd }}$ ed. Prentice Hall, New Jersey. 